\title{
Design of a Control Allocation Solution for the Winged Reusable Launch Vehicle ReFEx
}

\author{
Jose Luis Redondo Gutierrez* and David Seelbinder ${ }^{\dagger}$ \\ German Aerospace Center (DLR), Bremen, D-28359, Germany \\ Johannes Robens ${ }^{*}$ and Paul Acquatella B. ${ }^{\S}$ \\ German Aerospace Center (DLR), Oberpfaffenhofen, D-82234, Germany
}

\begin{abstract}
This paper presents a control allocation solution for the technology demonstrator mission ReFEx, which focuses on a vertical takeoff and horizontal landing strategy with autonomous navigation, online guidance, and controlled flight throughout the mission. The trajectory for the demonstration flight is aimed as one for a winged launch vehicle first stage: maintaining stability and control of the vehicle while reaching a predefined target. During the atmospheric phase the vehicle is stabilized by using an active aerodynamic control system which transforms inputs from the guidance and navigation systems into control commands for the individual actuators. In that sense, the control allocation subsystem translates commanded moments into commanded aerodynamic surface deflections. Due to the effect of modeling uncertainties, navigation errors, and underactuated regions, this subsystem needs to be robustified. The algorithm proposed in this paper addresses this challenge via a combination of the deflections required to trim the vehicle together with delta-deflections that aim at converging iteratively to the commanded moments. The combination of these two contributions is able to respond fast to state changes, compensate for modeling uncertainties and navigation errors, and provide a safe mode for the underactuated regions. The performance of the system is studied using a high-fidelity simulator.
\end{abstract}

\section{Nomenclature}

$\begin{array}{ll}\alpha, \beta, \mu & =\text { angle of attack, angle of sideslip, bank angle } \\ \omega & =\text { angular rate of the body frame w.r.t the inertial frame } \\ \bar{q} & =\text { dynamic pressure } \\ I & \text { moment of inertia } \\ C_{l}, C_{m}, C_{n} & =\text { aerodynamic coefficients for roll, pitch and yaw moments } \\ C & =\text { aerodynamic coefficients vector, }\left[C_{l}, C_{m}, C_{n}\right]^{T} \\ \mathrm{Ma} & =\text { Mach } \\ \eta_{S}, \eta_{A} & =\text { symmetric and asymmetric deflection of the canards } \\ \zeta & =\text { rudder deflection } \\ \delta & =\text { deflection vector, }\left[\eta_{S}, \eta_{A}, \zeta\right]^{T} \\ f & =\text { generic function }\end{array}$

\section{Introduction}

The complexity and cost of launch vehicles makes them a major target when aiming at reducing the expensiveness of access to space. Reusing these vehicles, either partly or completely, is among the strategies that are currently studied and implemented to reduce the share of the launch in the total cost of the mission. The German Aerospace Center (DLR) is currently developing several concepts for reusable launch vehicles, including both VTVL (Vertical Take-off Vertical

\footnotetext{
* GNC Engineer, Institute of Space Systems

${ }^{\dagger}$ Head of Guidance \& Control Group, Institute of Space Systems

${ }^{\ddagger}$ Control Engineer, Institute of System Dynamics and Control

${ }^{\S}$ Control Engineer, Institute of System Dynamics and Control
} 
Landing), such as CALLISTO [1], and VTHL (Vertical Take-off Horizontal Landing), such as ReFEx (Reusability Flight Experiment). This paper focuses on the latter.

ReFEx is part of the DLR effort to develop future winged reusable launcher stages and vehicles with reentry capabilities. The mission focuses on a vertical takeoff and horizontal landing (VTHL) strategy with autonomous navigation, online guidance, and controlled flight during each phase of the vehicle's mission with a demonstration flight that is planned for 2023 from the Koonibba Test Range in Australia. ReFEx does not use a main thruster to lower its kinetic energy during the reentry phase, but depends solely on its aerodynamic properties. Similarly, a RCS (Reaction Control System) is used to control the attitude of the vehicle only in the outer layers of the atmosphere, while aerodynamic actuators, i.e. canards and rudder, are used during the major part of the reentry flight. This paper focuses on the design and performance of the control allocation system used for the aerodynamic actuators, i.e. the translation of torques commanded by the control system to deflection signals to be forwarded to these actuators.

There are several challenges that arise from the sole use of aerodynamic actuators to control the vehicle, including: 1) the effect of modeling uncertainties, regarding vehicle's aerodynamics and actuators, and 2) the error in the estimated state, notably that in angle of attack, angle of sideslip and Mach. Certain peculiarities of ReFEx aerodynamics, such as the presence of underactuated regions, pose an additional test to the performance and robustness of the system.

The control allocation solution proposed in this paper addresses these challenges using an algorithm that computes in parallel two contributions to the deflections of the actuators: 1) a 'base' contribution, that aims at trimming the vehicle based on the estimated state and a precomputed database, and 2) a 'delta' contribution that aims at iteratively converging to the commanded torque, modifying the deflections based on the estimated torque. Due to the 'base' component the system responds fast to changes in the estimated state. This component also provides a safe mode to deal with underactuated regions. On the other hand, the 'delta' contribution is able to compensate for the biases that appear due to modelling uncertainties and navigation errors. This hybrid solution for the control allocation problem is the main contribution of this paper.

Section [I] contains a brief description of the mission ReFEx, including an overview of the vehicle, the trajectory to be flown and the different control allocation modes. In the following two sections, the control allocation problem is formulated [II and the particular difficulties solving this problem are described IV] In section $\mathrm{V}$ the proposed algorithm is explained in detail. Lastly, in section VI the performance of the system is studied using a high-fidelity simulator that includes realistic models of the vehicle dynamics, sensors, and actuators, as well as external disturbances and parametric uncertainties together with the GNC system in the loop.

\section{Mission}

ReFEx is a demonstrator mission that succeeds the DLR SHEFEX (Sharp Edge Flight Experiment) II, which was launched in 2012 [2]. However, SHEFEX II did not have a guidance system or significant lifting surfaces, in contrast to in ReFEx. ReFEx aims at performing an autonomously controlled flight, following a trajectory representative for a winged RLV (Reusable Launch Vehicle) first stage. Therefore the vehicle will transition from hypersonic speeds down to a subsonic regime.

This section introduces the vehicle and the trajectory for the mission, as well as the control allocation modes that are implemented. A more complete overview of the mission can be found in [3] [4].

\section{A. Vehicle}

The Re-Entry segment of ReFEx has approx. $400 \mathrm{~kg}$ of mass and a longitudinal length of $2.7 \mathrm{~m}$. The wingspan is $1.1 \mathrm{~m}$ and the diagonal terms of the moment of inertia are approx. $15 \mathrm{kgm}^{2}$ for the longitudinal axis and $240 \mathrm{kgm}^{2}$ for the other two axes. A section view of the vehicle is shown in figure 1.

Several iterations on the shape of the vehicle have been undertaken through the history of the mission in order to fulfill aerodynamic requirements for the planned range of velocities, i.e. from hypersonic to subsonic. The current design consists of two fixed wings located at the back of the vehicle, a fin with an attached vertical tail actuator (rudder) and two canards positioned close to the nose of the vehicle. The implications of this aerodynamic configuration are further analyzed in section IV.C.

As the translational motion is controlled by aerodynamic forces, the actuators are used only for controlling the attitude of the vehicle. Two sets of actuators are used: 1) RCS, with 8 thrusters located at the back of the vehicle, and 2) aerodynamic actuators, with the canards and rudder previously mentioned. The RCS thrusters have two thrust modes, generating 2.3 and $11.6 \mathrm{~N}$ respectively. The deflections of the aerodynamic actuators are mechanically limited to -20 and $20 \mathrm{deg}$. 


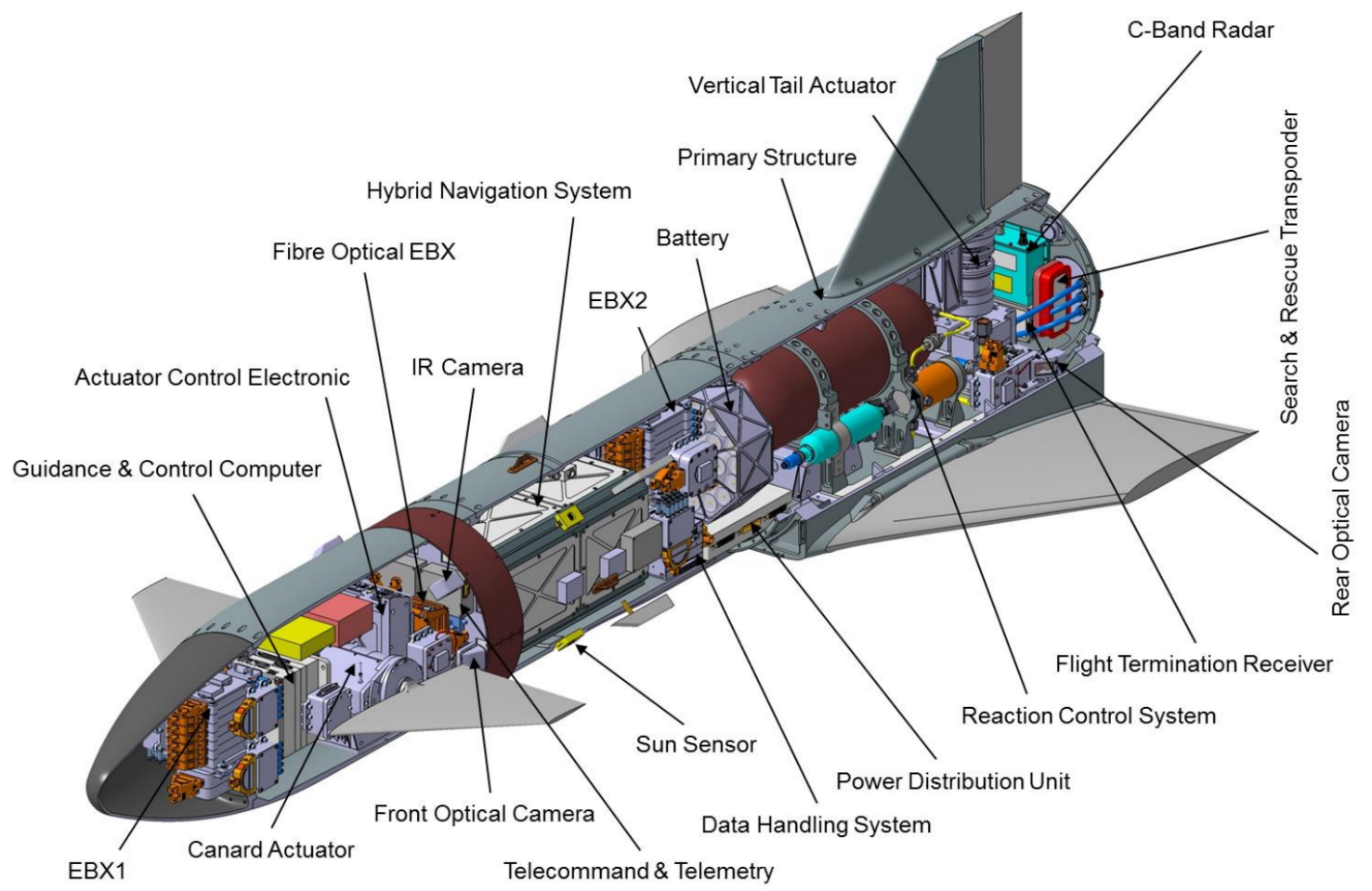

Fig. 1 Section view of the Re-Entry segment of ReFEx [3].

\section{B. Trajectory}

Figure 2 shows the sequence of events for the mission with some preliminary details. Several phases can be identified from this figure: 1) launch phase, 2) experimental phase until EI (Entry Interface), 3) experimental phase between EI and EoE (End of Experiment), and 4) experimental phase after EoE.

A Brazilian solid propellant two-stage rocket VSB-30 will be used during the launch phase. This rocket is unguided and only passively stabilized, leading to a considerable state uncertainty at separation. During this phase most of the Re-Entry segment is covered using a hammerhead fairing to minimize its aerodynamic effect. After the two stages are burned out, the rocket is spun down using a Yo-Yo system. Afterwards both fairing and rocket are separated. The current launch site is the Koonibba test range in Australia.

After separation and until EI the atmosphere is not dense enough to meaningfully affect the motion of the vehicle, leading to a ballistic trajectory. The aerodynamic actuators are also not effective and, thus, the RCS is used. The objectives during this phase are: 1) to detumble the vehicle after separation, 2) to acquire the sun to enable a convergence of the navigation solution, and 3) to reach EI with the desired attitude.

When EI is reached the dynamic pressure is already high enough to: 1) control the attitude of the vehicle using the aerodynamic actuators and 2) generate aerodynamic forces able to influence the trajectory. During this phase the objective is to use the aerodynamic forces to correct the trajectory, compensating the state dispersion at separation as well as other uncertainties. The target point to be reached at EoE is previously defined. After EoE the vehicle aims at dissipating as much energy as possible to minimize the ground impact.

\section{Control allocation modes}

The task of the control allocation is to define a command to the actuators such that the torques requested by the controller are generated as precisely as possible. In parallel to the presence of two sets of actuators, i.e. RCS and aerodynamic actuators, two control allocation modes are defined.

The RCS actuators are used from separation until the EI is reached. The rationale for this is that the aerodynamic actuators are not effective in the outer regions of the atmosphere due to a lack of dynamic pressure. Since the Entry Interface is defined by the dynamic pressure, its altitude varies depending on the velocity profile of the vehicle and the density-altitude curve. 




Fig. 2 Mission architecture \& flight events [3].

While using the RCS actuators, the control allocation strategy consists of the following steps: 1) define a set of cost functions penalizing the torque error and the fuel consumption, 2) transcribe the problem into a linear programming form and 3) solve it using a 2-Steps SIMPLEX method. The design of this control allocation mode will not be further explained in this paper. For further explanations on this methodology the reader is referred to [5].

When the dynamic pressure threshold, defined as $1000 \mathrm{~Pa}$, is reached, the control allocation switches to a second mode, which aims at generating the commanded torque by deflecting the aerodynamic actuators. This phase of the flight presents considerable challenges which have been met with innovative solutions. In the remainder of the paper we will focus first on the challenges that arose, then on the solution proposed and finally on the performance attained.

\section{Problem Formulation}

This paper presents a control allocation system intended for reentry vehicles in which aerodynamic surfaces are used to control the attitude motion. This section introduces the control allocation problem. The objective of the the control allocation subsystem is to generate a commanded torque using the aerodynamic control surfaces. This subsystem uses the information provided by the navigation subsystem to define a command to the actuators such that the torque commanded by the control subsystem is exerted over the vehicle (Fig. 3 ).

In ReFEx the commanded torque is defined using a cascaded (time-scale separation based) nonlinear dynamic inversion (NDI) control architecture [6]. This architecture exploits feedback linearization and linear control loops to decouple and partially linearize the attitude motion of the vehicle, using state feedback and the inversion of the attitude dynamic equation [7]

$$
\dot{\omega}=I^{-1}(T-\omega \times I \omega)
$$

where $\omega=\left[\begin{array}{lll}p & q & r\end{array}\right]^{T}$ is the angular rate of the body frame with respect to the inertial frame, expressed in 


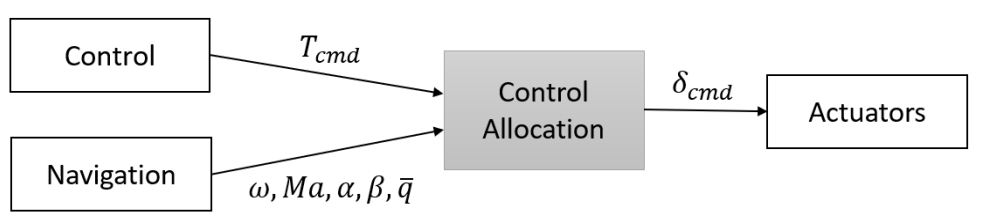

Fig. 3 Control allocation interfaces.

components along the body axes; $T=\left[\begin{array}{lll}T_{x} & T_{y} & T_{z}\end{array}\right]^{T}$ is the sum of external, Coriolis and relative torques about the center of mass, expressed in components along the body axes; and $I$ is the inertia tensor of the vehicle, referred to the body frame. The commanded torque is then defined as

$$
T_{\mathrm{cmd}}=I v_{\mathrm{des}}+\omega \times I \omega
$$

where $v_{\text {des }}$ represent the desired or reference angular accelerations obtained by the outer loop of the NDI architecture by means of linear (robust) control. In other words

$$
T_{\mathrm{cmd}}=I\left[\begin{array}{c}
\dot{p}_{\mathrm{des}} \\
\dot{q}_{\mathrm{des}} \\
\dot{r}_{\mathrm{des}}
\end{array}\right]+\left[\begin{array}{c}
p \\
q \\
r
\end{array}\right] \times I\left[\begin{array}{c}
p \\
q \\
r
\end{array}\right]
$$

Notice that in this setup, the commanded moments are being computed based on the desired dynamics behaviour of the closed-loop system. This is followed by the control allocation, which maps the commanded moments to the required deflections, which is the goal of this paper. In another setup, the inversion-based control architecture could also employ the aerodynamic control effectiveness in the formulation of the feedback linearization as proposed in [8, 9] and where the robustification of the controller is done via incremental nonlinear dynamic inversion (INDI). The current setup is a preliminary design, and in the future this control architecture is sought to be modified.

Once the commanded torque has been defined it is necessary to ensure its mapping into deflections by means of control allocation. As a simplification, the control allocation assumes that the commanded torque will be solely generated by the aerodynamics of the vehicle, i.e. all other contributions are not accounted for. The aerodynamic contribution to the torque $\left(T_{\text {aero }}\right)$ can be written as:

$$
T_{\text {aero }}=\bar{q} S_{\text {ref }} l_{\text {ref }}\left[\begin{array}{c}
C_{l} \\
C_{m} \\
C_{n}
\end{array}\right]
$$

where $\bar{q}$ is the dynamic pressure, $S_{\text {ref }}$ is the (aerodynamic) reference area, and $l_{\text {ref }}$ is the (aerodynamic) reference length. Moreover, we can represent the dependencies of these coefficients as

$$
\left[\begin{array}{c}
C_{l} \\
C_{m} \\
C_{n}
\end{array}\right]=\left[\begin{array}{c}
C_{l}\left(\mathrm{Ma}, \alpha, \beta, \eta_{S}, \eta_{A}, \zeta, \omega\right) \\
C_{m}\left(\mathrm{Ma}, \alpha, \beta, \eta_{S}, \eta_{A}, \zeta, \omega\right) \\
C_{n}\left(\mathrm{Ma}, \alpha, \beta, \eta_{S}, \eta_{A}, \zeta, \omega\right)
\end{array}\right]=f(\mathrm{Ma}, \alpha, \beta, \delta, \omega)
$$

where symmetric and asymmetric deflections of the canards $\left(\eta_{S}, \eta_{A}\right)$ are expressed as

$$
\left[\begin{array}{c}
\eta_{S} \\
\eta_{A}
\end{array}\right]=\left[\begin{array}{l}
\left(\eta_{R}+\eta_{L}\right) / 2 \\
\left(\eta_{R}-\eta_{L}\right) / 2
\end{array}\right]
$$

where $\eta_{R}$ and $\eta_{L}$ are the deflections of the right and left canards.

The control allocation problem consists of the inversion of both (4) and (5) in order to define a vector of commanded deflections $\delta_{\mathrm{cmd}}=\left[\eta_{S}, \eta_{\mathrm{A}}, \zeta\right]^{T}$ such that the commanded torques $T_{\mathrm{cmd}}=\left[L_{\mathrm{cmd}}, M_{\mathrm{cmd}}, N_{\mathrm{cmd}}\right]^{T}$ are exerted over the system. In other words, the control allocation problem consists of finding solutions to the inverse problem

$$
\delta_{\mathrm{cmd}}=f^{-1}\left(\mathrm{Ma}, \alpha, \beta, \omega, L_{\mathrm{cmd}}, M_{\mathrm{cmd}}, N_{\mathrm{cmd}}, \bar{q}\right)
$$




\section{Challenges}

Using aerodynamic actuators to control the attitude of an aircraft presents several challenges, which are considerably more complex for reentry vehicles. In ReFEx only aerodynamic actuators are used for the major part of the mission. This is a critical aspect of the mission and drives the need of developing a reliant and efficacious system. This section describes these challenges, which are (among others): 1) state estimation error, 2) modeling uncertainties and actuator dynamics and 3) particularities of the aerodynamics of the vehicle.

\section{A. State estimation error}

To find the deflections that generate a commanded torque, two steps are required: 1) to calculate the aerodynamic coefficients and 2) to compute the deflections $\left(\eta_{S}, \eta_{A}, \zeta\right)$ that would generate those coefficients.

The first step is based on (4). It can be observed that the error in $\bar{q}$ is incorporated in the aerodynamic coefficients. The second step consists of defining the relation between the desired coefficients and the related deflections (5). These coefficients do not only depend on the deflections of the actuators but rather on a considerably extensive set of state variables. All of these variables will have estimation errors, which will then be transferred to that relation.

Even though each of the variables presented in (4) and (5) has an effect on the final discrepancy between commanded and actual torque, it was found that, for ReFEx, the most critical ones are $\alpha$ and $\beta$. This is due to the huge effect of changes in these variables in the aerodynamic coefficients. The error in the estimation of these variables is driven by: 1) the error in the attitude with respect to the local NED (North East Down reference frame) and 2) the wind. Of these, the latter contributes considerably more to the final error. Several wind analyses, conducted taking into account ReFEx launching site and trajectory, pointed towards unbearable uncertainties of more than $10 \mathrm{deg}$ in both variables, when the wind was not accounted for in the navigation algorithms. As a result, a FADS (Flush Air Data System) was integrated into the vehicle, to be able to estimate local aerodynamic angles directly. Despite the introduction of this novel sensor, the estimation of $\alpha$ and $\beta$ is still subject to considerable errors, particularly before reaching the $\bar{q}$ threshold needed for the sensor to function adequately.

\section{B. Modeling uncertainties and actuator dynamics}

The modeling uncertainties considered for the current analyses of the mission are related to: initial state, atmospheric parameters, mass properties, aerodynamic coefficients, actuators alignment, wind profile and sensors. Out of these the uncertainties in aerodynamic coefficients and actuators alignment have a direct impact on the control allocation.

The aerodynamic coefficients are computed by superposition of the effect of different variables, as shown in (8). This equation is an extended version of (5). Each of these terms incorporates an uncertainty and, therefore, the relation between the state of the vehicle, including deflections, and the aerodynamic coefficients has an error in itself.

$$
C=C\left(M a, \alpha, \eta_{S}\right)+C_{\beta}(M a, \alpha, \beta)+C_{\eta_{A}}\left(M a, \alpha, \eta_{A}\right)+C_{\zeta}(M a, \alpha, \zeta)+C_{\omega}(M a, \alpha, \omega)
$$

The errors in the actuators alignment lead to a constant bias between the commanded deflections and the actual ones. Besides these misalignments, there are two aspects of the actuators dynamics that need to be considered when designing a control allocation system: 1) the dynamic transients and 2) the presence of a deadband. Even though the actuator dynamics are considerably fast, they still limit the reaction time. The deadband results in a minimum angular variation that can be commanded. This deadband is a tool to account for the expected uncertainty and has no physical meaning. Due to the high operating frequency of the actuators, the actual and commanded deflections are always separated by the magnitude of the deadband.

\section{Aerodynamics}

The analysis presented here is conducted over an aerodynamic database generated for ReFEx by the Institute of Aerodynamics and Flow Technology, also part of DLR, using CFD (Computational Fluid Mechanics) simulations. More information about the generation of this database and its validation can be found in [10] and [11]. The aerodynamic coefficients can be extracted from this database using (8). Throughout this subsection some aerodynamic particularities of the vehicle are studied, emphasizing their impact on the control allocation. It is important to keep in mind that these analyses are conducted over the nominal aerodynamic database and, thus, do not incorporate the effect of uncertainties in the aerodynamic coefficients.

First of all, the regions of Ma, $\alpha$ and $\beta$ where the vehicle can be trimmed are found. Trimmability is here defined as the presence of a set of canard and rudder deflections resulting in a negligible aerodynamic torque. This analysis is 


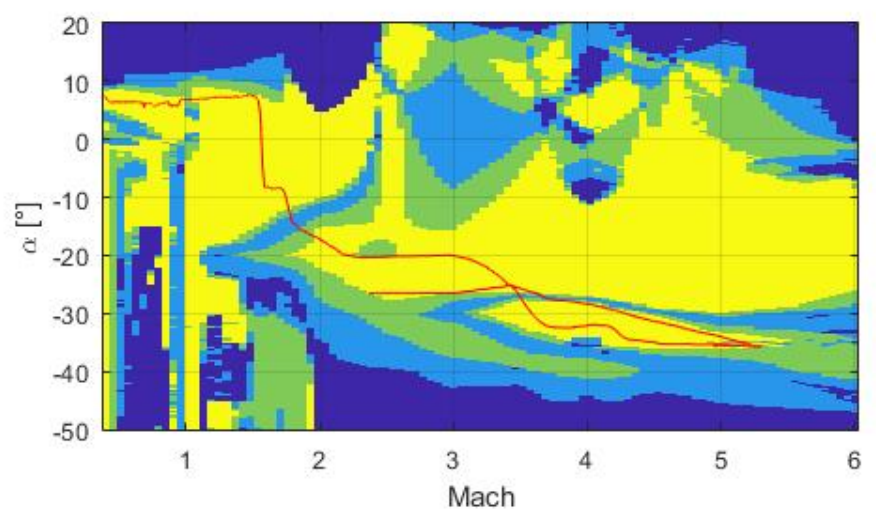

Fig. 4 Trimmability analysis. In yellow the region trimmable for $|\beta| \leq 2 \mathrm{deg}$, in green for $|\beta| \leq 1 \mathrm{deg}$ and in light blue for $\beta=0$. In red the nominal trajectory.

conducted over a 3-dimensional grid of Ma, $\alpha$ and $\beta$. In each point of the grid the deflections that minimize the cost function

$$
F_{C}=k_{1}\left|C_{l}\right|+k_{2}\left|C_{m}\right|+k_{3}\left|C_{n}\right|
$$

where $k_{1}, k_{2}$ and $k_{3}$ are positive constants. This optimization is executed using the MATLAB optimization toolbox and takes into account the limitations on the deflections of the actuators. Figure 4 shows the results of this analysis.

The results show that the vehicle is trimmable for an extensive combination of Ma and $\alpha$ when $\beta$ is zero. However, as soon as $\beta$ starts to increase untrimmable regions appear not only at the border of the previously trimmable region, but also inside of it. These inner untrimmable regions grow as $\beta$ increases, quickly become divisive lines between the regions where the vehicle can be trimmed. Due to the requirements of the mission (see nominal trajectory) it is clear that the vehicle has to cross these lines at least twice. Therefore, it is necessary to understand what is the root of this phenomenon. To do so, $\beta$ is fixed at $1 \mathrm{deg}$ and the aerodynamic coefficients resulting from trying to trim the vehicle are studied (Fig. 5).

Figure 5 shows that minimizing $C_{m}$ presents less difficulties that reducing $C_{l}$ and $C_{n}$. Furthermore, it can be observed that the regions where $C_{l}$ and $C_{n}$ are high are extremely similar to each other and to the untrimmable regions shown in fig. 4. Three conclusions can be drawn from these results: 1) $C_{m}$ can be trimmed (almost) independently, 2) $C_{l}$ and $C_{n}$ are highly coupled, and 3 ) the untrimmable regions are caused by the impossibility to simultaneously reduce $C_{l}$ and $C_{n}$ in those regions.

Figure 6 analyses the effectiveness matrix, i.e. the matrix containing the partial derivatives of the coefficients with respect to the deflections. These results confirm that $C_{m}$ is relatively independent and can be controlled via $\eta_{S}$. It also shows that $C_{l}$ and $C_{n}$ depend on $\eta_{A}$ and $\zeta$, thus they are coupled. Particularly interesting is the evolution of the term that contains the effect of $\eta_{A}$ in $C_{l}$ (top center of fig. 6). When this term is small the roll torque that can be applied by $\eta_{A}$ is extremely limited, leading to an underactuated vehicle. As it is not possible to reduce both $C_{l}$ and $C_{n}$ using $\zeta$, the vehicle is untrimmable. Furthermore, $\partial C_{l} / \partial \eta_{A}$ has different signs on each side of the regions where it is small. This sign change is a potential source of instabilities, as sudden control reversals in $\eta_{A}$ would be needed to maintain the same $C_{l}$.

\section{Control Allocation Design}

As explained in section [III] the aim of the control allocation system is to find a set of deflections for the aerodynamic actuators that exerts the commanded torques over the system. Furthermore, the system needs to be robust against the different uncertainties and system particularities presented in section IV.

Ideally the design of the control allocation would consist of a one-to-one relation between desired torques and current state on one side and deflections of the aerodynamic actuators on the other side. While this could theoretically be done by defining a function $f$ such that it fulfills equation (7), it would not result in a viable option for ReFEx. The combined effect of state estimation errors, modeling uncertainties, actuator dynamics and aerodynamic particularities of the system, cannot be neglected without making the system less robust. 

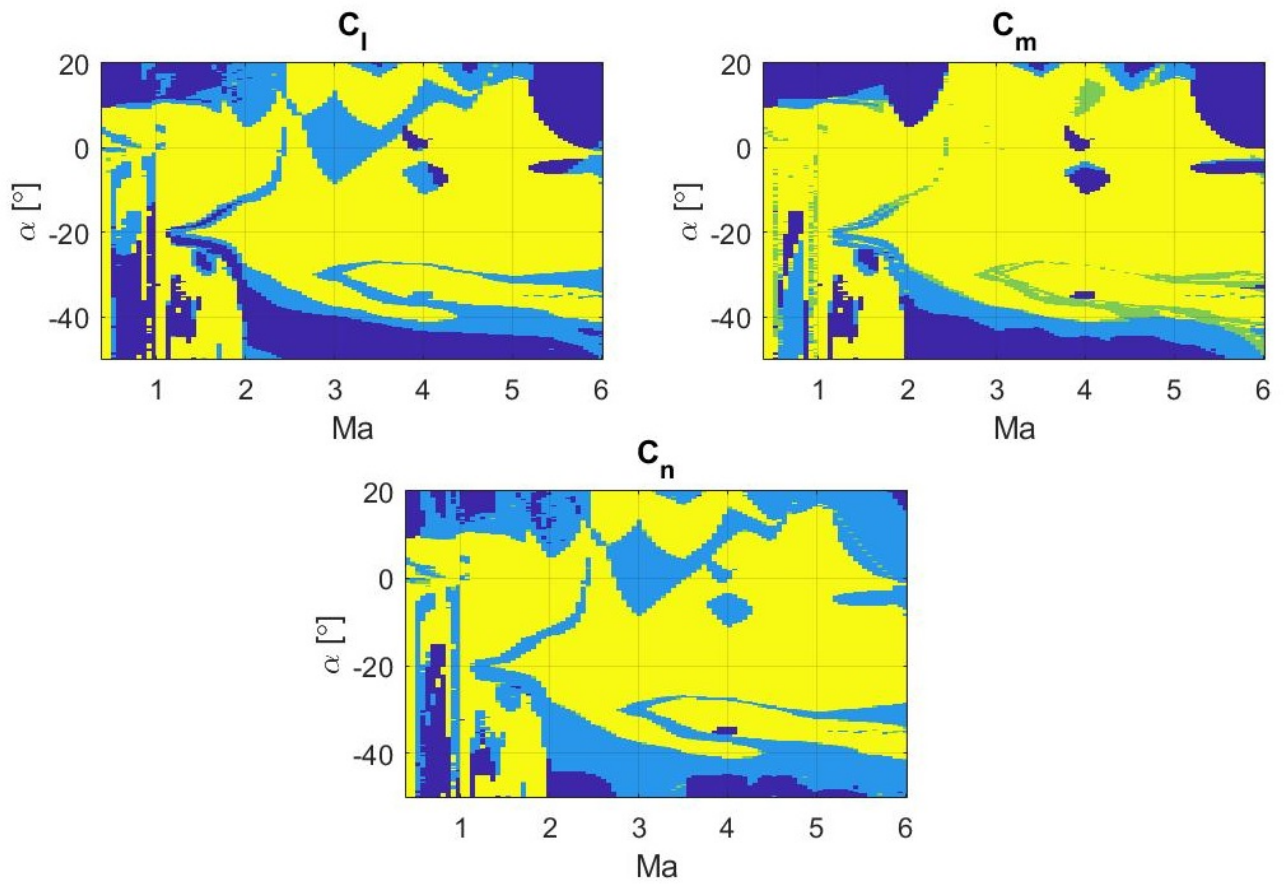

Fig. $5 C_{l}, C_{m}$ and $C_{n}$ resulting from trimming the vehicle at $\beta=1 \mathrm{deg}$. In yellow the region with a coefficient value $\leq 1 \mathrm{e}-6$, in green for $\leq 1 \mathrm{e}-4$ and in light blue for $\leq 1 \mathrm{e}-2$.

The remainder of this section proposes a control allocation solution that robustifies the system while complying with the performance requirements of the mission. In order to realize this objective, an algorithm with integral action using state feedback is defined, combining: 1) off-line optimization of the trimmability conditions, and 2) linearization of the control effectiveness of the aerodynamic actuators. This algorithm is further enhanced by taking into account vehicle limitations such as the location of the underactuated regions.

\section{A. Algorithm}

The control allocation algorithm relies on an integral action, that constantly adjusts the deflections of the aerodynamic actuators to generate the desired torque, reducing the impact of uncertainties and errors. The top level design of the control allocation is shown in figure 7. There are mainly two contributions to the deflections commanded: 1$) \delta_{\text {trim }}$, to trim the vehicle according to the estimated navigation state, and 2) $\Delta \delta$, to generate the commanded torque. Both steps are based on the results presented in section IV.C regarding trimmability and effectiveness. The calculation of these two contributions is further described in the rest of the subsection.

The 'base' deflections $\left(\delta_{\text {trim }}\right)$ aim at minimizing the produced torque, by either trimming the vehicle or applying the minimum possible torque. They are precomputed for a 3-dimensional grid in Ma, $\alpha$ and $\beta$. For this purpose the effect of $\omega$ on the coefficients is neglected. This leads to a 3-dimensional database that contains the relation between Ma, $\alpha$ and $\beta$ and $\eta_{S}, \eta_{A}$ and $\zeta$, that would minimize the generated torque. The final trim deflections are linearly interpolated from the database using the estimated state.

$$
\delta_{\text {trim }}=\left[\begin{array}{c}
\eta_{S} \\
\eta_{A} \\
\zeta
\end{array}\right]_{\text {trim }}=f_{\text {trim }}(M a, \alpha, \beta)
$$

The computation of the 'delta' deflections $(\Delta \delta)$ is considerably more complex. First of all, the current torque is estimated using the estimated angular rates as 

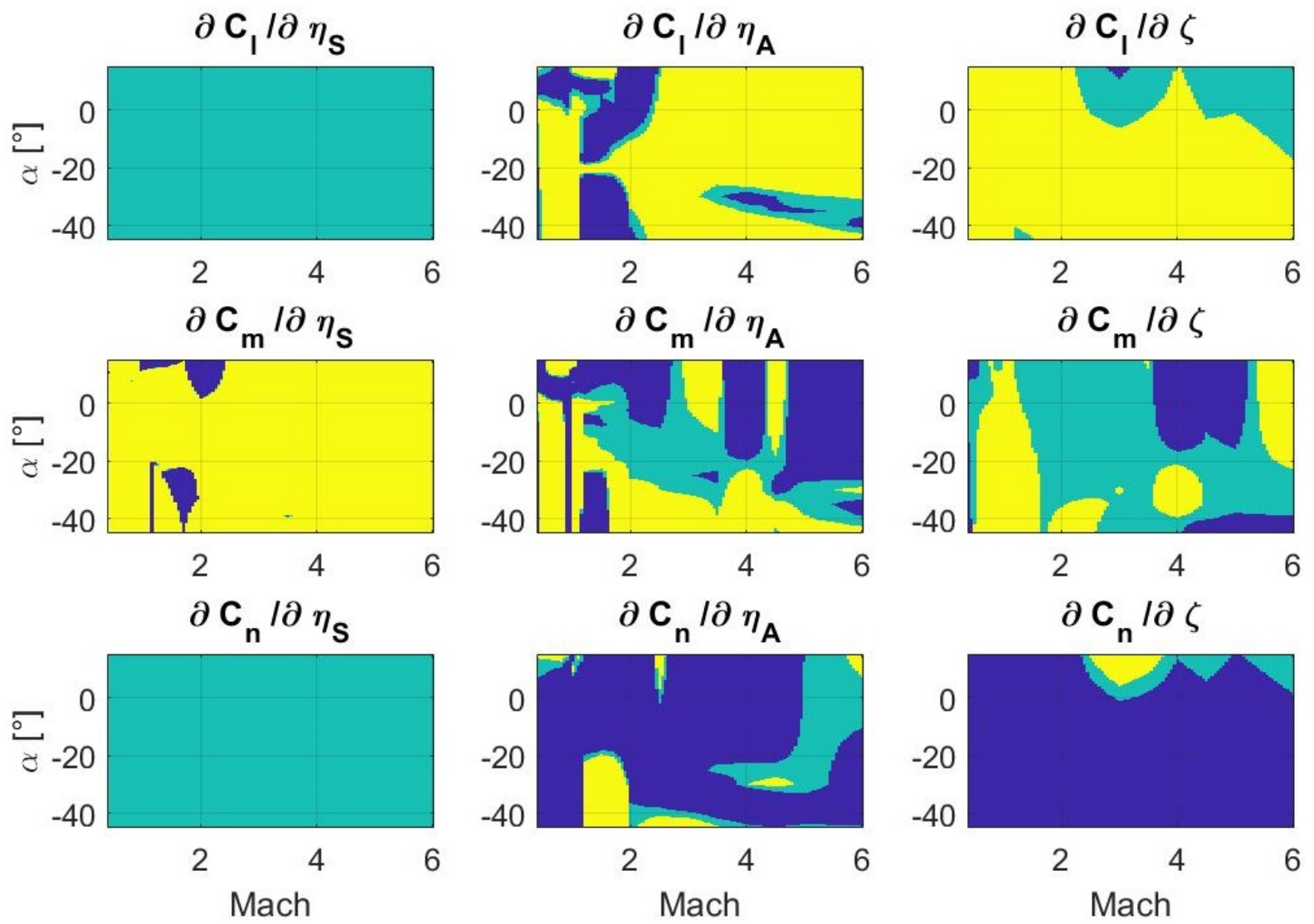

Fig. 6 Effectiveness analysis. In yellow the region with value $>5 e-4$, in green with value between $-5 e-4$ and $5 e-4$, and in blue $<-5 e-4$. 


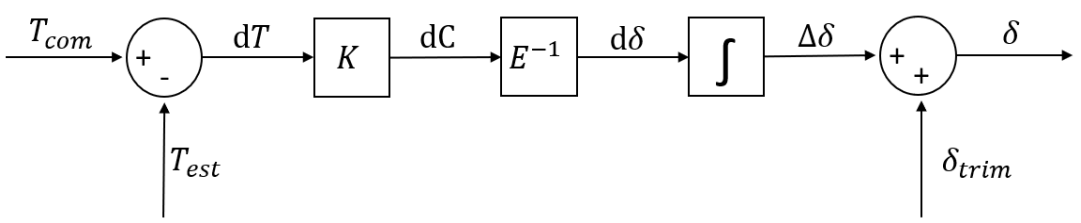

Fig. 7 Control allocation strategy.

$$
\begin{aligned}
\dot{\omega} & =\frac{\omega_{t}-\omega_{t-1}}{d t} \\
T_{\text {est }} & =I \dot{\omega}+\omega \times(I \omega)
\end{aligned}
$$

It is foreseen that this estimation will be conducted using more sophisticated filters in the future. After computing the difference between the estimated and commanded torque $(d T)$, it is converted into a difference in aerodynamic coefficients $(d C)$, using (4). Therefore, $K$ in fig. 7 is defined as

$$
K=\frac{1}{\bar{q} S_{\text {ref }} l_{\text {ref }}}
$$

In order to generate the desired difference in the aerodynamic coefficients, i.e. compensating for $d C$, the effectiveness matrix $(E)$ is used. This matrix contains the partial derivatives of the aerodynamic coefficients $\left(C_{l}, C_{m}, C_{n}\right)$ w.r.t. the deflections of the aerodynamic coefficients $\left(\eta_{S}, \eta_{A}, \zeta\right)$. These partial derivatives are computed at the trimmed state and are a function of Ma, $\alpha, \eta_{A}$ and $\zeta$. The dependency on the last two is limited to the terms $\frac{\partial C_{m}}{\partial \eta_{A}}$ and $\frac{\partial C_{m}}{\partial \zeta}$, and is caused by the symmetry of the vehicle. These partial derivatives are assumed to be constant, effectively linearizing the system in a similar manner as proposed in [8].

$$
E=\left[\begin{array}{lll}
\frac{\partial C_{l}}{\partial \eta_{S}} & \frac{\partial C_{l}}{\partial \eta_{A}} & \frac{\partial C_{l}}{\partial \zeta} \\
\frac{\partial C_{m}}{\partial \eta_{S}} & \frac{\partial C_{m}}{\partial \eta_{A}} & \frac{\partial C_{m}}{\partial \zeta} \\
\frac{\partial C_{n}}{\partial \eta_{S}} & \frac{\partial C_{n}}{\partial \eta_{A}} & \frac{\partial C_{n}}{\partial \zeta}
\end{array}\right]=F\left(M a, \alpha, \eta_{A}, \zeta\right)
$$

By inverting the matrix $E$ the change in the deflections that would be needed to generate the desired change in the coefficients can be obtained.

$$
d \delta=d\left[\begin{array}{c}
\eta_{S} \\
\eta_{A} \\
\zeta
\end{array}\right]=E^{-1} d C
$$

After both contributions for the deflections, 'base' and 'delta', have been obtained they are combined as shown in (13). There are additional limitations imposed over this expression, which are explained in the next subsection.

$$
\delta=\delta_{\text {trim }}+\int d \delta=\delta_{\text {trim }}+\Delta \delta
$$

This algorithm is built in such a way that it is able to: 1) react fast to changes of Ma, $\alpha$ and $\beta$ due to the trimmed deflections, and 2) counteract the effect of the errors and uncertainties shown in section IV] due to the integrated component of the deflections. The next section explains the particularities of the implementation and the degrees of freedom that the designer has when implementing the algorithm.

\section{B. Implementation}

This subsection describes the practical aspects to be considered when implementing the algorithm explained in the previous subsection. The implementation focuses on ensuring stability of the system and convergence to the commanded torque.

Aiming at correcting the torque instantaneously, i.e. changing the deflections in the manner proposed in (12), results in an extremely fast and unstable system. This is expected both from a control theory perspective and from the analysis 
of the system, as the algorithm would aim at reaching the desired torque within one time step. When factoring in the uncertainties of the system it becomes clear that the robustness of such an approach is minimal and that a 'slower' iterative solution needs to be implemented to guarantee convergence and stability. Therefore, (12) is transformed into

$$
d \delta=E^{-1}(k d C)
$$

where $k$ is a diagonal square matrix with all diagonal terms being positive constants smaller than 1 . The particular value of each term is tuned addressing the particularities of the dynamics of the system and the mission requirements. In ReFEx, the coupling between $C_{l}$ and $C_{n}$ due to their mutual dependencies in $\eta_{A}$ and $\zeta$ makes it necessary to set considerably lower gains for those coefficients than for $C_{m}$. The gains defined have an impact on the aggressiveness with which the commanded torque is reached, acting as a control loop. This offers a way to tune the algorithm to search for optimal performance under different requirements.

The effect of imposing saturation limits to the values of $d \delta$ and $\Delta \delta$ is also studied. To avoid continuously commanding extremely fast changes in the deflections, risking exciting an oscillatory behaviour on the commanded deflections, a maximum change in deflections per time step is specified. This parameter is not necessarily the same for the different deflections. A mathematical expression for this saturation is provided in (15).

$$
d \delta_{s a t, i}= \begin{cases}d \delta_{\max , i}, & \text { if } d \delta_{i}>d \delta_{\max , i} \\ d \delta_{\min , i}, & \text { if } d \delta_{i}<d \delta_{\min , i} \\ d \delta_{i}, & \text { otherwise }\end{cases}
$$

The saturation of $\Delta \delta$, i.e. $\int d \delta$, is originated from two main necessities: 1) to stay within the range of deflections of the actuators and 2) to avoid diverging in excess from the trimmed state. In relation to the former, dynamic saturation functions $(F)$ are defined, taking into account the trimmed deflections and the maximum allowed deflections (16). To realize the second point, an absolute saturation limit is specified (17).

$$
\begin{gathered}
\Delta \eta_{\text {Smax }}=F\left(\eta_{\text {Strim }}, \eta_{\text {Atrim }}, \eta_{\text {max }}\right) \\
\Delta \zeta_{\text {max }}=F\left(\zeta_{\text {trim }}, \zeta_{\text {max }}\right) \\
\Delta \eta_{\text {Amax }}=F\left(\eta_{\text {Strim }}, \Delta \eta_{S}, \eta_{\text {Atrim }}, \eta_{\text {max }}\right) \\
\Delta \delta_{\text {sat }, i}= \begin{cases}\Delta \delta_{\text {max }, i}, & \text { if } \int d \delta_{\text {sat }, i}>\Delta \delta_{\max , i} \\
-\Delta \delta_{\text {max }, i}, & \text { if } \int d \delta_{\text {sat }, i}<-\Delta \delta_{\text {max }, i} \\
\int d \delta_{\text {sat }, i}, & \text { otherwise }\end{cases}
\end{gathered}
$$

Additionally, the underactuated regions (see section IV.C) have a major impact on the control allocation as well, thus they have to be addressed in the implementation of the algorithm. When going through these regions, the effectiveness matrix $(E)$ becomes singular or close to singular. As a result, it is impossible to generate certain desired torques by deflecting the aerodynamic actuators. Additionally, the effect of some actuator deflections, particularly the effect of $\eta_{A}$ in $C_{l}$, can have opposite sign on each side of the underactuated region (see figure 6).

In order to address these two phenomena a control allocation safe mode is defined. This mode is entered when the effectiveness matrix becomes close to singular, as this coincides with an underactuated region. When the system switches to the safe mode the integration of the 'delta' deflections (see (13) ) is reset to zero, thus only commanding the trim deflections. This avoids an oscillatory behaviour with high amplitude in the commanded deflections while aiming at maintaining a minimum torque. Therefore, the trim deflections are not only used to react to fast changes of Ma, $\alpha$ and $\beta$, but also as the baseline of the safe mode. While this safe mode reduces the inherent instability that appears when flying in underactuated regions, it will diverge in the long term due to the uncertainties mentioned in section IV.B. Thus, it is of necessary to minimize the time spent in those regions.

Two aspects of the algorithm are still challenging. The first one is the occurrence of rapid changes in the trim values. These can be triggered by sudden changes in the state estimation or crossings of the control reversal regions. As the trim deflections are directly fed forward (fig. 7), sudden changes on their values would translate in rapid changes in the commanded deflections. Secondly, $d \delta$ is computed as the addition to the commanded deflections at the previous time step, that is needed to generate the commanded torque. However, as the trim deflections also change in each time step, an additional change in the commanded deflections is added. Even though this additional change is in general negligible, it can play a considerable role in the proximity of underactuated regions. 


\section{Performance}

This section focuses on the performance of the control allocation design proposed in section $\mathrm{V}$. Additionally it assesses the individual impact of the challenges mentioned in section IV

The performance is analyzed by using two simulation setups, with 3 and 6 DoF (Degrees of Freedom). For the $3-\mathrm{DoF}$ analysis the vehicle's position and velocity are fixed and only the rotational dynamics are simulated. The 6-DoF setup simulates the actual trajectory of the mission. Both analyses contain the same models for external disturbances, actuators, sensors and control systems. The main differences in the modeling are:

- For the 3-DoF analysis the guidance signal consists of predefined ramp commands in the aerodynamic angles ( $\alpha$, $\beta$ and $\mu$ ), while the 6-DoF setup integrates the guidance software in the loop. In the latter the guidance modifies the reference trajectory at every timestep based on the current navigation solution in order to reach the target point at EoE.

- For the 3-DoF analysis the error of the navigation solution is modelled with random-walk and noise, mimicking the behaviour of the actual navigation system. In the 6-DoF analysis the navigation system is also integrated in the loop.

The results presented for both simulation setups are:

- Evolution of estimated $\alpha, \beta$ and $\mu$. These results provide an overall understanding of the behaviour of the system analyzed.

- Evolution of commanded and generated torques, reflecting the precision of the control allocation system.

- Evolution of the commanded deflections, in order to understand the nature of the control allocation command.

\section{A. 3-DoF Analysis}

As previously mentioned, this analysis simulates only the rotational dynamics of the vehicle, keeping position and velocity fixed at a point on the nominal trajectory. As the nominal trajectory goes through considerably different regions (e.g. positive and negative $\alpha$ ), three points were initially selected for the analysis. As these three points show extremely similar behaviour, only one of them was included in this paper for the sake of brevity.

The guidance output consists of predefined ramp commands in $\alpha, \beta$ and $\mu$. Figure 8 shows the evolution of the control and navigation errors for $\alpha, \beta$ and $\mu$ under these commands. The simulation setup used for the analysis shown in figure 8 contains all errors and uncertainties previously described.

The control error remains small during most of the simulation, except when big angular changes are commanded (e.g. $\mu$ at $t=100 s$ ). Therefore, the control allocation system is able to compensate for the estimation and modeling errors. It is also noticeable that the control errors show an oscillatory/noisy behaviour. This behaviour is mainly caused by: 1) the error in the navigation solution and 2) the deadband in the actuators. In order to further understand the effect of each error source (see section IV the behaviour of the system is studied under 5 different assumptions: 1) nominal plant, 2) with actuator uncertainties, 3) with uncertainties in the aerodynamic coefficients, 4) with navigation errors and 5) actual plant. Figure 9 shows the evolution of the commanded torque and the error of the torque for each of these cases in an interval of $10 \mathrm{~s}$. This figure contains the same maneuvers as figure 8 but shows a shorter time interval in order to enable a clearer analysis.

For the nominal plant as well as for only considering aerodynamic uncertainties the commanded torque and its error are quickly driven to zero. Even though aerodynamic uncertainties seem to have almost no effect, it has to be considered that as Ma and $\alpha$ do not change suddenly, the error in these coefficients is almost constant. Therefore, the conclusion is that the control allocation is able to handle constant biases in the aerodynamic coefficients, rather than that errors in these coefficients have a negligible effect. The actuator uncertainties and dynamics prevent the system from exactly reaching the commanded deflections and, thus, there is always a residual torque command. This is also the case when only navigation errors are considered, even though these have a stronger effect. Nevertheless, even for the actual plant, the realized and the commanded torque are close enough to each other to produce the desired attitude, as it can be seen in Fig. 8

Figure 10 shows the evolution of the commanded deflections for the same maneuvers as the analysis in figure 8 . comparing them for nominal and actual plant. The difference between these deflections can be divided in 3 contributions: 1) fast oscillations, 2) slow oscillations and 3) biases. Fast oscillations are those with frequencies higher than $1 \mathrm{~Hz}$ and are a consequence of the actuator dynamics, the noise in the estimated state and the aggressiveness of the control algorithms. The slow oscillations, with frequencies of around $0.1 \mathrm{~Hz}$, are caused by slow changes in the state estimation error. Finally, the biases appear due to the actuator misalignments and the aerodynamic uncertainties. 

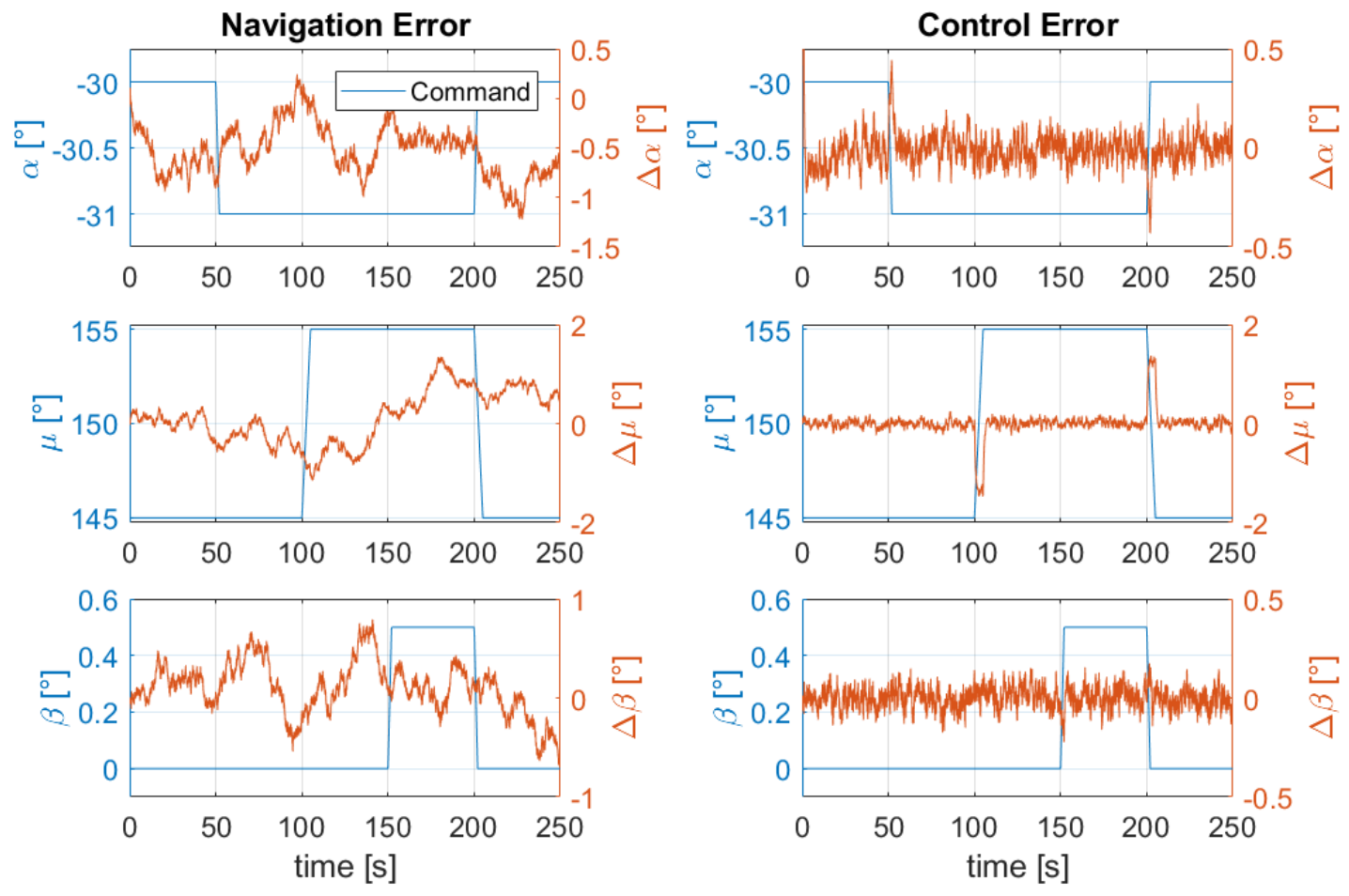

Fig. 8 Evolution of $\alpha, \beta$ and $\mu$ under ramp commands.
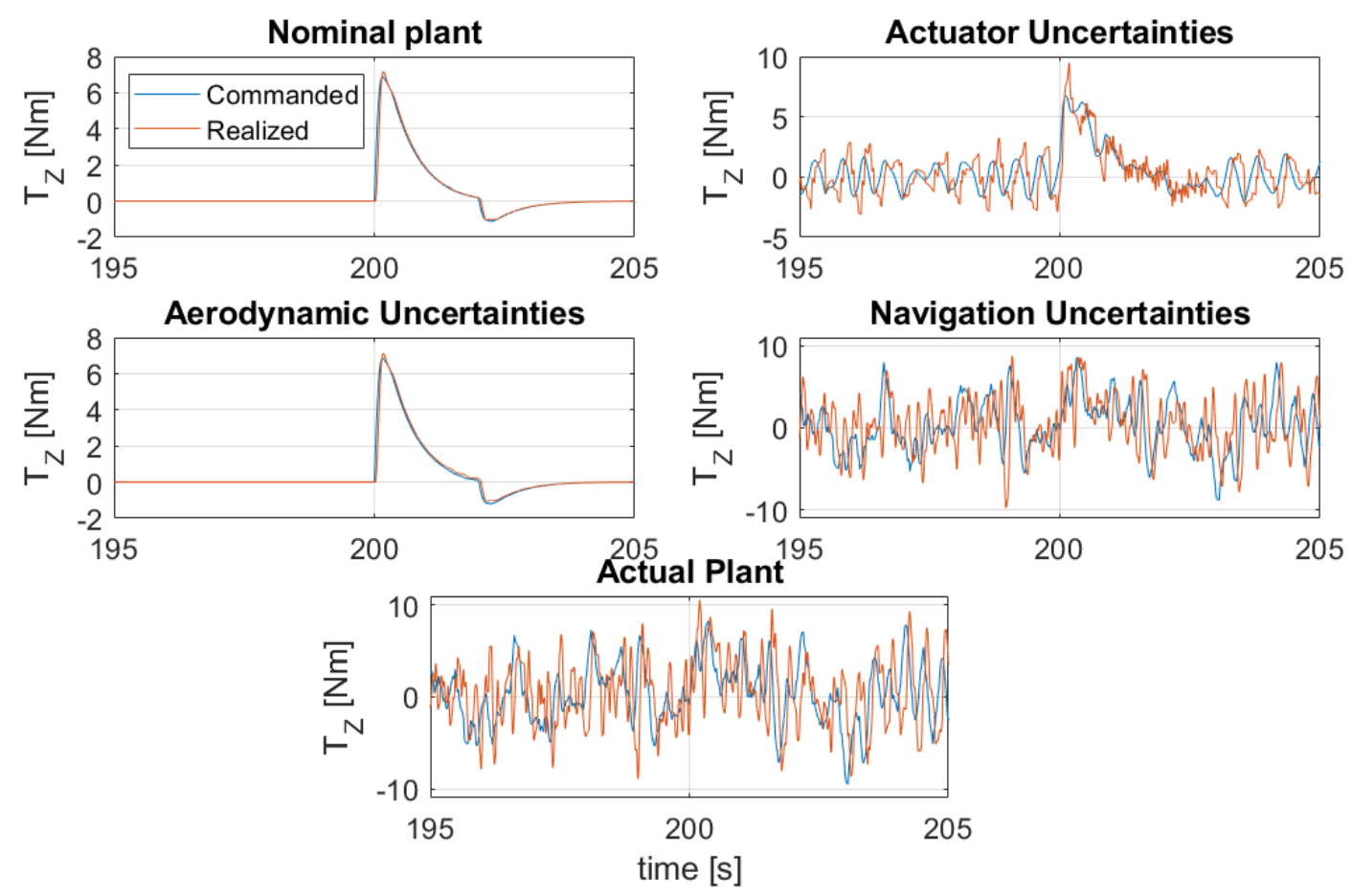

Fig. 9 Evolution of commanded torque and torque error. 

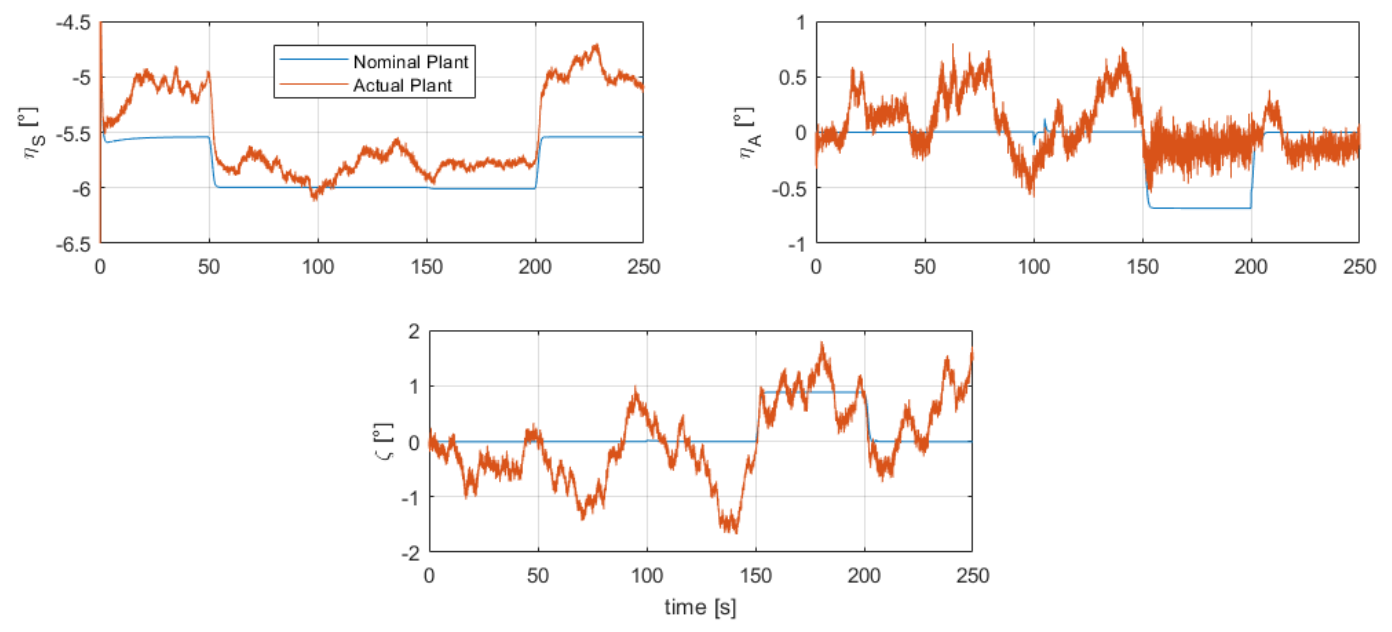

Fig. 10 Evolution of commanded deflections.

\section{B. 6-DoF analysis}

In this subsection similar analyses as those in subsectionVI.A are conducted, but the 6-DoF setup and a simulated reentry trajectory are used. Therefore, position and velocity are also propagated. The analyses focus on the evolution of the vehicle between a point closely after it reaches the apogee of the trajectory to a point after the EoE is reached, which is at around $9 \mathrm{~km}$ altitude. Even though the following results could also be used to draw conclusions regarding the performance of other subsystems, such as control or guidance, the content of this paper will remain focus solely on what directly affects the control allocation.

First of all, the evolution of the commanded aerodynamic angles as well as their control and estimation errors are presented in figure 11. Launch is assumed to be at $t=0$, thus the investigated interval starts at a later point in time. It can be observed that the control error is considerably higher than in the 3-DoF analysis. This increase is driven mainly by: 1) faster changes in the commanded angles, 2) crossing underactuated regions and 3) limitations in actuator effectiveness due to low dynamic pressure at the beginning of the reentry. The first point is particularly noticeable for the roll maneuver, which inverts $\mu$, that takes place around $t=400 \mathrm{~s}$ in the simulated trajectory. The vehicle crosses the underactuated regions (see section IV.C) around $t=365 \mathrm{~s}$ and $t=390 \mathrm{~s}$, with a consequent increase of the error, particularly in $\beta$. The navigation error is considerably reduced as the vehicle dives into the lower layers of the atmosphere.

Secondly, the commanded and generated torques are studied. In figure 12 the evolution of these two variables is studied for two time intervals of the trajectory. The first interval, on the left, focuses on the roll maneuver, and the second one on a representative part of the rest of the trajectory. During the roll maneuver the control allocation is able to follow the commanded torque considerably accurately, with a small delay due to the integral action of the algorithm. In the second interval the commanded torque is one order of magnitude smaller, as no maneuver is conducted. As a result, then noise to signal ratio is higher in both commanded and generate torques.

Figure 13 shows on one side the torque error, i.e. the difference between commanded and realized torque, and on the other side the deflections of the actuators. When crossing the underactuated regions (around $t=365 \mathrm{~s}$ and $t=390 \mathrm{~s}$ ), peaks in the torque error occur. This is caused by triggering the safe mode, which aims at trimming the vehicle instead of exerting the commanded torque. Crossing these regions also has an impact on the deflections of the actuators, which become: 1) larger due to the lower effectiveness, which can potentially compromise the total loads on the actuators, and 2) noisier due to the fast sign changes of terms in the effectiveness matrix, which can increase the deflection errors as the actuators are not able to follow the evolution of the command accurately. In addition, it can be observed that $\eta_{S}$ changes to trim the vehicle at the actual angle of attack.

The analysis shows that the control allocation design is able to overcome the several challenges presented in section [V] generating the desired torques while maintaining the vehicle stable during the flight. 

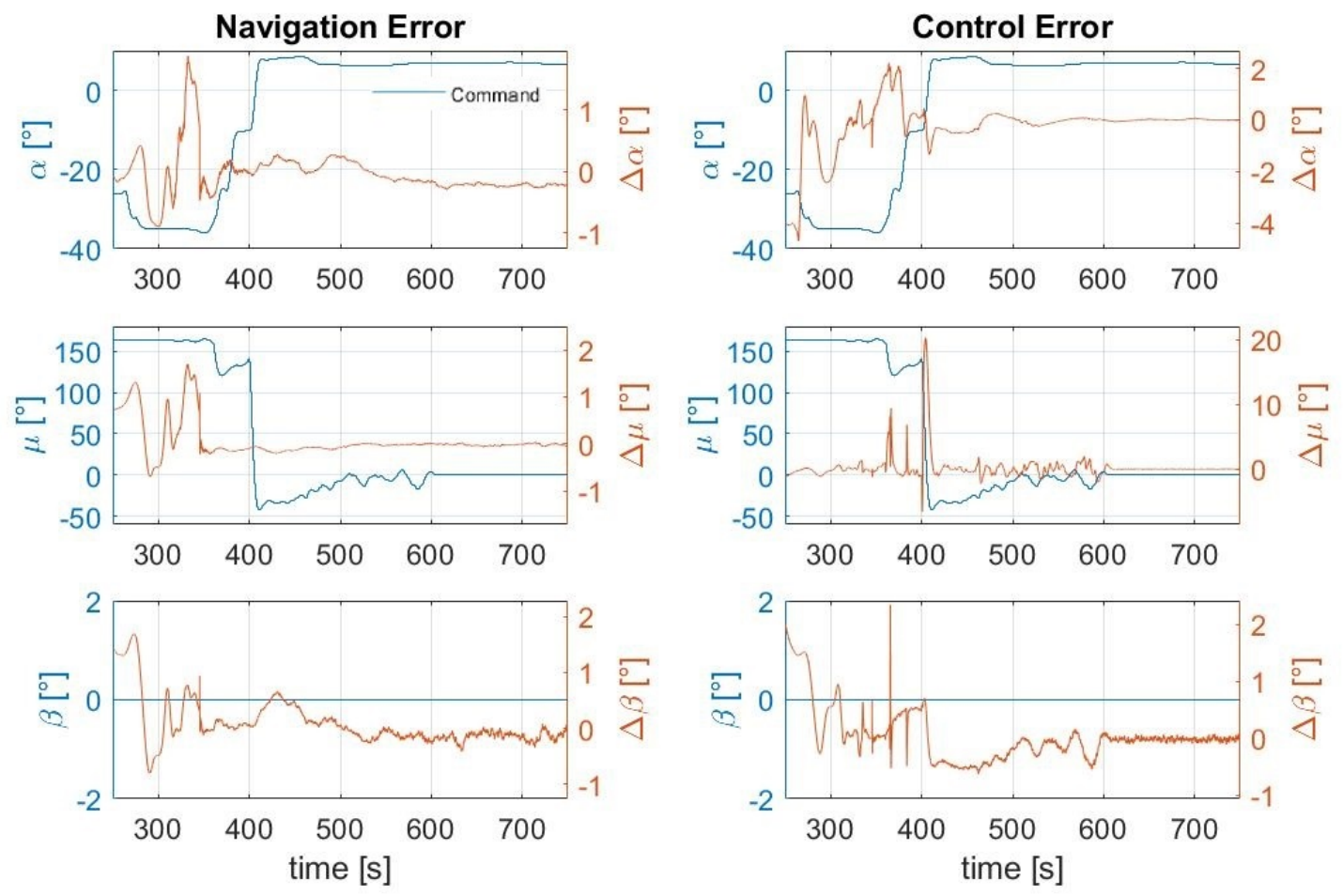

Fig. 11 Evolution of $\alpha, \beta$ and $\mu$ and their navigation and control errors over a sample trajectory.
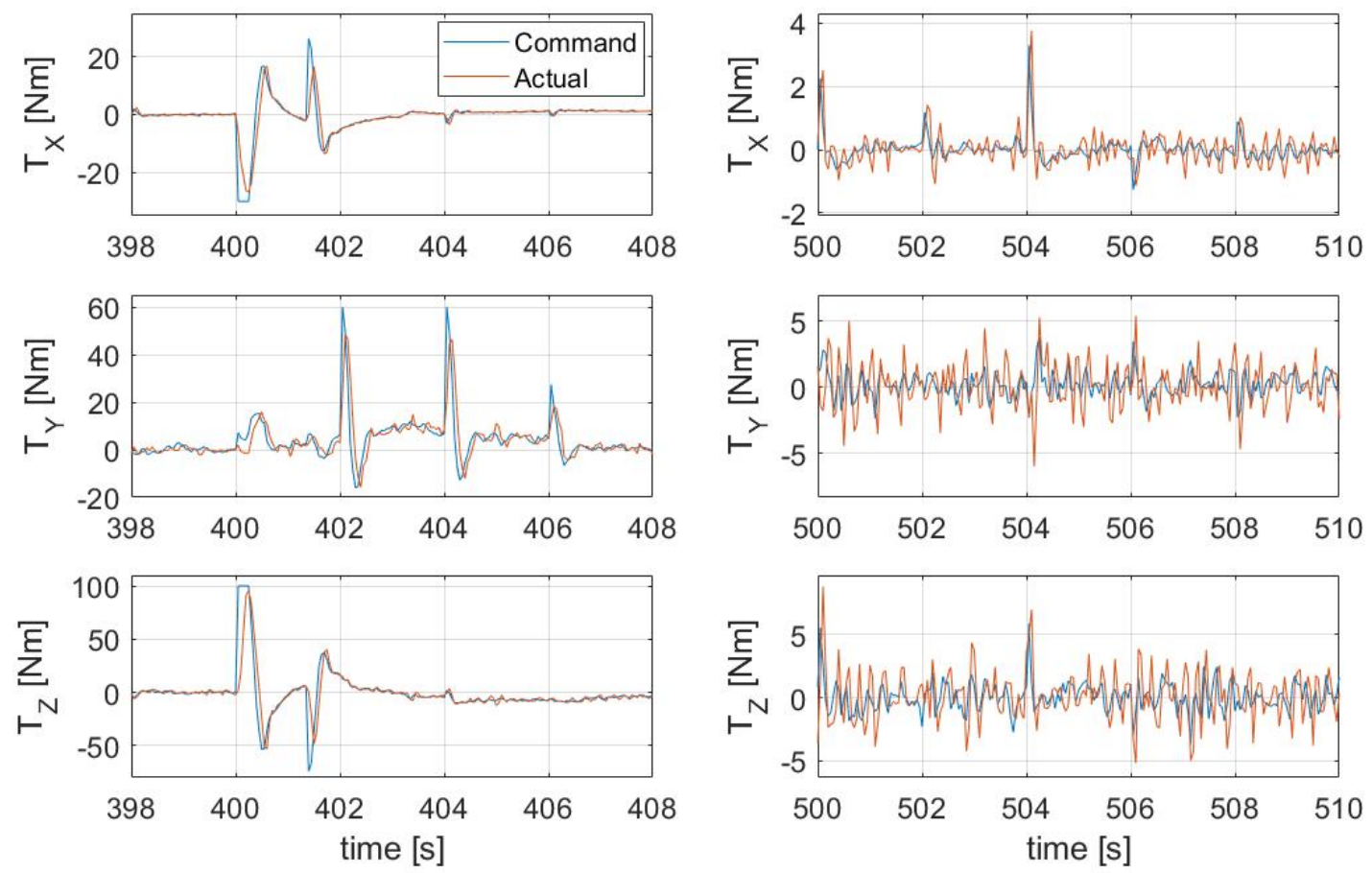

Fig. 12 Evolution of commanded and actual torque for two time intervals. 

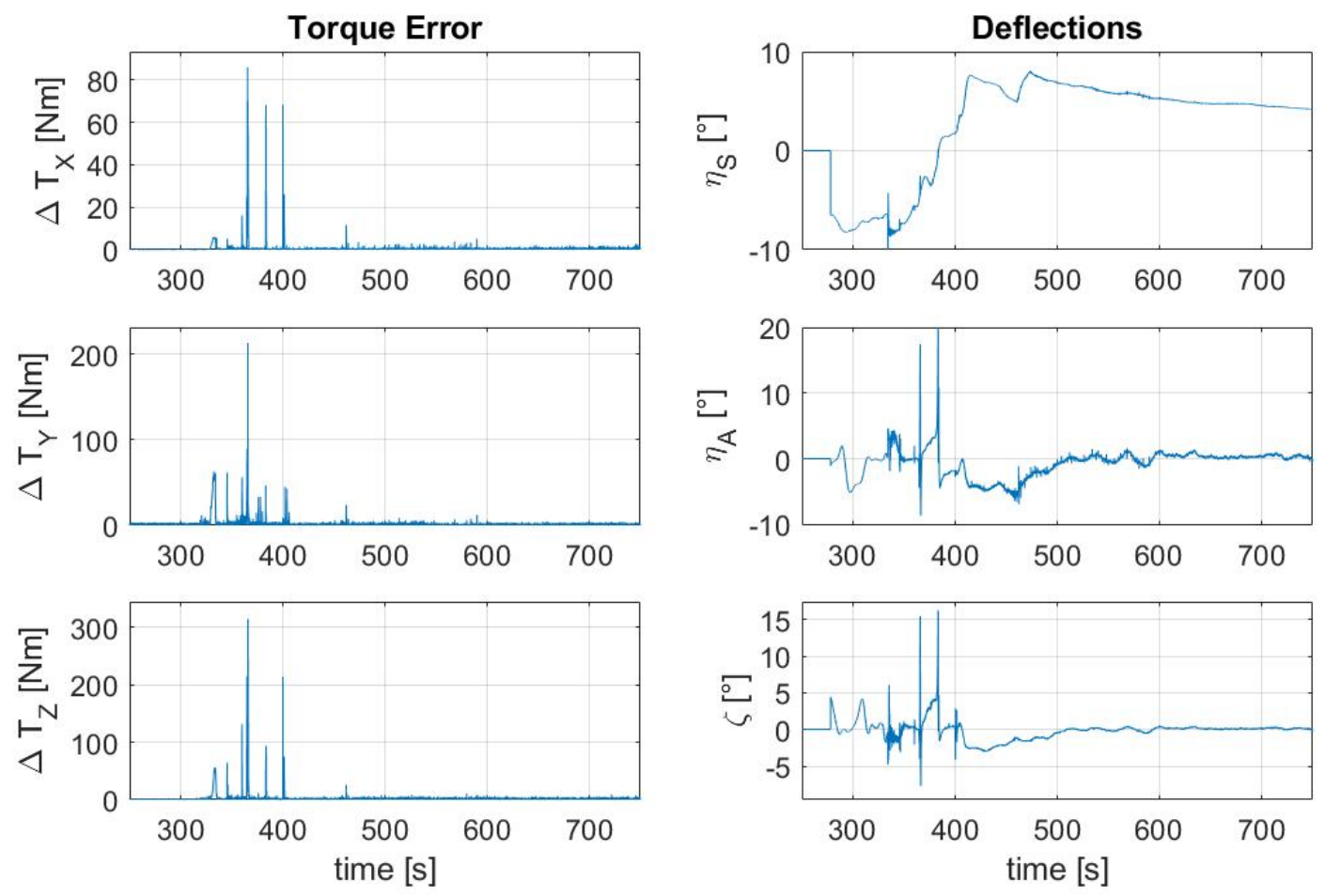

Fig. 13 Evolution of the error in the generated torque and of the actuator deflections

\section{Conclusion}

The objective of this paper was to present the design and performance of a control allocation strategy for the aerodynamic control surfaces of the DLR demonstrator mission ReFEx during its atmospheric flight phase.

The control allocation strategy for ReFEx is particularly challenging because of the effect of state estimation errors, model and parametric uncertainties, and the nonlinear dynamics of the vehicle. The underactuated regions where controllability and effectivenes are partially lost are particularly challenging. Typical control allocation algorithms coordinate the effectors (deflections) such that they produce the desired control efforts (moments) whenever possible, which is usually done by a static mapping. The control allocation algorithm proposed in this paper combines 'base' deflections (static) and 'delta' deflections (dynamic) to determine the total deflection command to the effectors (the actuator of the aerodynamic surfaces). The 'base' deflections depend only on the estimated state of the vehicle and are thus considered in open loop; they provide the deflections that would generate the minimum possible torque at the estimated state assuming a perfect knowledge of the vehicle model. The 'delta' deflections aim at dynamically minimizing the difference between the estimated and commanded moments, therefore their computation can be seen as an internal closed-loop control. The combination of these two contributions leads to a system that: (1) is able to react fast to changes in the estimated state (base deflections), (2) is able to converge to a desired moment command despite constant or slow-changing errors (delta deflections) and (3) has a relatively stable safe mode that enables the vehicle to fly through underactuated regions where controllability and effectiveness are partially lost.

The performance of the control allocation system along with the tuning of its parameters is studied for ReFEx by conducting analyses in 3-DoF (attitude) and 6-DoF simulations. The 3-DoF attitude analysis is used to study the effect of different uncertainties on the vehicle behaviour and points to the navigation error as the dominant factor. The analysis over the complete trajectory in 6-DoF simulations shows that, despite the noticeable effect of going through underactuated regions, the proposed algorithm is able to overcome the mission challenges and with its integration in the flight control chain, it helps to fulfill the mission according to systems-level requirements. 


\section{References}

[1] Schwarz, R., Solari, M., Razgus, B., Dumke, M., Markgraf, M., Bestard Körner, M., Pfau, D., Reigenborn, M., Braun, B., and Sommer, J., "Preliminary Design of the Hybrid Navigation System (HNS) for the CALLISTO RLV Demonstrator," $8 t h$ European Conference for Aeronautics and Space Sciences (EUCASS), 2019.

[2] Eggers, T., "The Shefex II experimental re-entry vehicle: Presentation of flight test results," 28th International Congress of the Aeronautical Science (ICAS), 2012.

[3] Bauer, W., Rickmers, P., Kallenbach, A., Stappert, S., Wartemann, V., Merrem, C. H.-J., Schwarz, R., Sagliano, M., Grundmann, J. T., Flock, A., et al., "DLR reusability flight experiment ReFEx," Acta Astronautica, Vol. 168, 2020, pp. 57-68.

[4] Rickmers, P., Bauer, W., Sippel, M., and Stappert, S., "ReFEx: Reusability Flight Experiment-A Flight Experiment to Demonstrate Controlled Aerodynamic Flight from Hypersonic to Subsonic Velocities with a Winged RLV," 7th European Conference for Aeronautics and Space Sciences (EUCASS), July, 2017, pp. 3-6.

[5] Nelder, J. A., and Mead, R., “A simplex method for function minimization,” The computer journal, Vol. 7, No. 4, 1965, pp. 308-313.

[6] Acquatella B., P., Briese, L. E., and Schnepper, K., "Guidance command generation and nonlinear dynamic inversion control for reusable launch vehicles," Acta Astronautica, Vol. 174, 2020, pp. 334-346. https://doi.org/https://doi.org/10.1016/j.actaastro. 2020.04 .002

[7] Stevens, B. L., Lewis, F. L., and Johnson, E. N., Aircraft control and simulation: dynamics, controls design, and autonomous systems, John Wiley \& Sons, 2015.

[8] Sieberling, S., Chu, Q. P., and Mulder, J. A., "Robust Flight Control Using Incremental Nonlinear Dynamic Inversion and Angular Acceleration Prediction," Journal of Guidance, Control and Dynamics, Vol. 33, No. 6, 2010, pp. $1732-1742$. https://doi.org/https://doi.org/10.2514/1.49978.

[9] P. Acquatella B., W. van Ekeren, Q.P. Chu, "PI(D) tuning for Flight Control Systems via Incremental Nonlinear Dynamic Inversion,” IFAC-PapersOnLine, Vol. 50, No. 1, 2017, pp. 8175 - 8180. https://doi.org/https://doi.org/10.1016/j.ifacol.2017.08. 1265, 20th IFAC World Congress.

[10] Merrem, C. H.-J., Wartemann, V., and Eggers, T., "Aerodynamic Data Set Generation for the Experimental Vehicle ReFEx," Notes on Numerical Fluid Mechanics and Multidisciplinary Design, 2021, pp. 132-140.

[11] Wartemann, V., Konosidou, N., Flock, A. K., and Merrem, C. H.-J., "Contribution of Numerical and Experimental Flow Simulations to the Aerodynamic Data Base of the DLR Reusable Flight Experiment ReFEx," Notes on Numerical Fluid Mechanics and Multidisciplinary Design, Vol. 151, 2021. 